\title{
Radiative efficiency of black holes in AGN
}

\author{
Dipo Mahto ${ }^{1}$, Rama Nand Mehta ${ }^{2}$, Umakant Prasad ${ }^{3}$, Raj Kumar Sah ${ }^{4}$, Krishna Murari Singh ${ }^{1}$ \\ ${ }^{1}$ Department of Physics, Marwari College, T.M.B.U. Bhagalpur-812007, India \\ ${ }^{2}$ Department of Mathematics, T.M.B.U. Bhagalpur-812007, India \\ ${ }^{3}$ Department of Physics, T. N. B. College, T.M.B.U. Bhagalpur-812007, India \\ ${ }^{4}$ Dept. of Physics, S.S.V. College, Kahalgaon, T.M.B.U. Bhagalpur-812007, India
}

\section{Email address:}

dipomahto@hotmail.com(D.Mahto),rnm1k@yahoo.co.in (R. N. Mehta), umakant.prasad@gmail.com (U. Prasad), dr.rajkumarsah@rediffmail.com (R. K. Sah),kmsinghphy@gmail.com (K. M. Singh)

\section{To cite this article:}

Dipo Mahto, Rama Nand Mehta, Umakant Prasad, Raj Kumar Sah, Krishna Murari Singh. Radiative Efficiency of Black Holes in AGN. International Journal of Astrophysics and Space Science. Vol. 1, No. 4, 2013, pp. 52-55. doi: 10.11648/j.ijass.20130104.16

\begin{abstract}
Neeraj Pant et al.(2010) gave a detailed study of BCT First solution (Tewari,1988) representing time dependent balls of perfect fluid with matter-radiation in general relativity to assume the life time of quasar $10^{7}$ years, initial mass $\approx 10^{8} M_{\Theta}$ with an initial linear dimension $\approx 10^{15} \mathrm{~cm}$ are radiating energy at a constant rate i.e. $L_{\infty}=10^{47} \mathrm{ergs} / \mathrm{sec}$ with the gravitational red shift, $\mathrm{z}=0.44637$. For quasars with $L_{Q S O} \sim 10^{46} \mathrm{erg} / \mathrm{sec}$., the black hole masses in those systems must be at least $10^{8}$ solar masses (Astro 3830: Spring 2004). In the present work, we have calculated the radiative efficiency of black holes existing in AGN by using the formula $L / \dot{M} c^{2}$ (Narayan2005, Xie \& Yuan 2012) and compared our results regarding some characteristics of super massive black holes with some other works given in references like Astro 3830:Spring (2004), Narayan (2005), Xie \& Yuan (2012) and Chan (2013) and concluded that the mass of black holes never be greater than $5 \times 10^{9} M_{\odot}$ as well as the radiative efficiency of black hole is smaller than the same mass of quasars.
\end{abstract}

Keywords: XRBs, Radiative Efficiency, AGN

\section{Introduction}

Galactic nuclei generally considered to be active have bolometric luminosities of $10^{-2}$ to $10^{4}$ times their host galaxy luminosity and are powered by accretion onto a super massive black hole. The fundamental parameters that determine accretion onto the central black hole should be: (a) the black hole mass (b) the almost of gas and dust in the galactic nucleus(c) a mechanism to derive material in the galactic nucleus onto the black hole and (d) outflows as a result of accretion, which may effect (b) and (c) as feedback (Mckernan et al, 2010).

There are three revealing common properties of AGNs as discussed given below: First, AGNs are extremely compact. Flux variability-a staple of all AGNs confines AGNs to within the distance light can travel in a typical variability timescale. In many cases, X-ray variability is observed on timescales of less than a day, and flares on time scales of minutes (e.g., MCG 6-30-15, McHardy, 1988). Second, the spectral energy distribution is decisively non stellar: roughly speaking, AGNs power per unit logarithmic frequency interval is constant over seven decades in frequency, while stars emit nearly all of their power in a frequency range a mere factor three wide. Third, AGNs must be very massive, a conclusion supported by two independent arguments. AGNs bolometric luminosities are astoundingly large: at least comparable, and often several orders of magnitude larger than the luminosity of entire surrounding galaxy. Masses in excess of nearly $10^{6} \mathrm{M}_{\odot}$ are needed for an AGN not to become unbound by its own outpouring of energy. Further more, according to our the best estimates, AGNs remain active for upward of $10^{7}$ years: during this period, an enormous amount of material, well over a million solar masses, must be consumed to sustain their luminosity, even assuming a very high efficiency of energy production (Ferrarese \& Ford, 2005).

Several AGNs and black hole X-ray binaries show a clear very broad iron line, which is strong evidence that the black hole are rapidly spinning. The strong gravitational light 
bending in these regions then explains the power-law variability as due to changes in the height of the primary $\mathrm{X}$-ray source above the disc. The reflection component, in particular its variability and the profile of the iron line, enables us to study the inner most regions around an accreting spinning black hole (Fabian, 2005).

Neeraj Pant et al. (2010) presented a model of BCT first solution keeping in view of conditions with most important phenomenon of immense gravity objects assuming the life time of quasar $10^{7}$ years, initial mass $\approx 10^{8} M_{\Theta}$ with an initial linear dimension $\approx 10^{15} \mathrm{~cm}$ that this model is radiating energy at a constant rate i.e. $L_{\infty} \approx 10^{47} \mathrm{ergs} / \mathrm{sec}$ ( Pant \& Tiwari, 2010).

In the present work, we have calculated the efficiency parameter (radiative efficiency) of black holes existing in AGN by using the formula $L / \dot{M} c^{2}$ (Narayan, $2005 \&$ Xie and Yuan, 2012) and compared our results regarding some characteristics of super massive black holes with some other works given in references like Astro 3830: Spring (2004), Narayan (2005), Pant et al. (2010), Xie \& Yuan (2012) and Chan (2013).

\section{Expression for Radiative Efficiency of Black Holes in AGN}

The radiative efficiency of an accretion disk is defined as the energy it radiates per unit accreted mass and hence it is given by (Narayan 2005, Xie and Yuan, 2012 \& Chan, 2013).

$$
\eta=L_{a c c} / \dot{M c^{2}}
$$

For non-rotating black hole, $\eta=0.057$, whereas a co-rotating disk around a maximally-rotating $\mathrm{BH}\left(a_{*}=1\right)$ has a much higher efficiency, $\eta=0.42$.

The radiative efficiency of an accretion disk in terms of the radius of the event horizon is given by

$$
\eta=\frac{G M_{B H} \dot{M}}{R_{b h}} / \dot{M c^{2}}
$$

where

$$
L=G M_{B H} \dot{M} / R_{b h}
$$

On the basis of the equation (2), the radiative efficiency $(\eta)$ of black holes for $R_{\text {stable }}=9 G M / c^{2}, 6 G M / c^{2}$, $2 G M / c^{2}, 1.2 G M / c^{2}$ and $G M / c^{2}$ are 0.11, 0.17, $0.50,0.83$ and 1.00 respectively (Astro 3830: Spring 2004).

\section{Data in Support of Mass of Black Holes in AGN}

There are two categories of black holes classified on the basis of their masses clearly very distinct from each other, with very different masses $\mathrm{M} \sim 5-20 \mathrm{M}_{\odot}$ for stellar - mass black holes in X-ray binaries and $\mathrm{M} \sim 10^{6}-10^{9.5} \mathrm{M}_{\odot}$ for super massive black holes in Active Galactic Nuclei (Narayan, 2005 \& Mahto et al. 2012) and masses in the range $10^{6} M_{\odot} t o 3 \times 10^{9.5} M_{\odot}$ have been estimated by this means in about 20 galaxies (Narayan, 2005). The other data in the support of the mass of black holes in AGN can be seen in the research paper (Mahto et al. 2013).

\section{Calculation}

The formula for Eddington Luminosity of a black hole of the mass $(\mathrm{M})$ is given by

$$
L_{E}=1.3 \times 10^{38}\left(\mathrm{M} / \mathrm{M}_{\odot}\right) \mathrm{erg} / \mathrm{sec} .
$$

$$
\text { For } M=10^{6} M_{\odot}, L . E=1.3 \times 10^{44} \mathrm{erg} / \mathrm{sec} \text {. }
$$

and other values of LE are listed in the table.

The Radiative efficiency of a black hole of the mass $(M)$ is given by following equation as:

$$
\eta=L_{E} / \dot{M c^{2}}
$$

where LE is the total luminosity emitted from the accretion flow and $\dot{M}$ is the corresponding mass accretion rate of the system (Xie and Yuan, 2012). The term $\dot{M}$ is defined as one solar mass per year (Astro 3830: Spring 2004) i.e.

$\dot{M}=$ One solar mass/one year.

$\dot{M}=\frac{2 \times 10^{31}}{31536} \mathrm{gram} / \mathrm{sec}$.

Hence

$$
\begin{aligned}
& \dot{M} c^{2}=\frac{2 \times 10^{31}}{31536}\left(3 \times 10^{10} \mathrm{~m} / \mathrm{s}\right)^{2} \mathrm{gram} / \mathrm{sec} . \\
& \dot{M} c^{2}=\frac{18 \times 10^{51}}{31536} \mathrm{gm}^{2} \mathrm{sec}^{3}
\end{aligned}
$$

The radiative efficiency $(\eta)$ of black holes of the mass $M=10^{6} M_{\odot}$ and Eddington Luminosity $=1.3 \times 1044 \mathrm{erg} / \mathrm{sec}$. may be calculated as:

$$
\eta=\frac{L_{E}}{\dot{M c^{2}}}=\frac{1.3 \times 10^{44}}{18 \times 10^{51}} \times 31536=\frac{1.3 \times 31536}{18 \times 10^{7}}=.0002
$$

Similarly, the radiative efficiency of other different black holes in AGN has been calculated in the following table. 
Table. Radiative efficiency of black holes in AGN

\begin{tabular}{|c|c|c|c|c|}
\hline $\begin{array}{l}\text { Sl. } \\
\text { No. }\end{array}$ & $\begin{array}{l}\text { Mass of } \\
\text { BHs (M) }\end{array}$ & $\begin{array}{l}\left(R_{b h}=2950 \mathrm{M} /\right. \\
\left.M_{\square}\right) \\
\text { (in metre) }\end{array}$ & $\begin{array}{l}\text { Eddington } \\
\text { Luminosity } \\
\left(L_{E}\right) \text { in } \\
\text { erg/s }\end{array}$ & $\begin{array}{l}\text { Radiative } \\
\text { efficiency }(\eta)\end{array}$ \\
\hline 1 & $1 \times 10^{6} M_{\odot}$ & $0.0295 \times 10^{11}$ & $1.3 \times 10^{44}$ & 0.00022 \\
\hline 2 & $2 \times 10^{6} M_{\odot}$ & $0.0595 \times 10^{11}$ & $2.6 \times 10^{44}$ & 0.00044 \\
\hline 3 & $3 \times 10^{6} M_{\odot}$ & $0.0885 \times 10^{11}$ & $3.9 \times 10^{44}$ & 0.00066 \\
\hline 4 & $4 \times 10^{6} M_{\odot}$ & $0.1180 \times 10^{11}$ & $5.2 \times 10^{44}$ & 0.00088 \\
\hline 5 & $5 \times 10^{6} M_{\odot}$ & $0.1475 \times 10^{11}$ & $6.5 \times 10^{44}$ & 0.00110 \\
\hline 6 & $6 \times 10^{6} M_{\odot}$ & $0.1770 \times 10^{11}$ & $7.8 \times 10^{44}$ & 0.00132 \\
\hline 7 & $7 \times 10^{6} M_{\odot}$ & $0.2065 \times 10^{11}$ & $9.1 \times 10^{44}$ & 0.00154 \\
\hline 8 & $8 \times 10^{6} M_{\odot}$ & $0.2360 \times 10^{11}$ & $10.4 \times 10^{44}$ & 0.00176 \\
\hline 9 & $9 \times 10^{6} M_{\odot}$ & $0.2655 \times 10^{11}$ & $11.7 \times 10^{44}$ & 0.00198 \\
\hline 10 & $1 \times 10^{7} M_{\odot}$ & $0.2950 \times 10^{11}$ & $1.3 \times 10^{45}$ & 0.00227 \\
\hline 11 & $2 \times 10^{7} M_{\odot}$ & $0.5950 \times 10^{11}$ & $2.6 \times 10^{45}$ & 0.00454 \\
\hline 12 & $3 \times 10^{7} M_{\odot}$ & $0.8850 \times 10^{11}$ & $3.9 \times 10^{45}$ & 0.00681 \\
\hline 13 & $4 \times 10^{7} M_{\odot}$ & $1.180 \times 10^{11}$ & $5.2 \times 10^{45}$ & 0.00908 \\
\hline 14 & $5 \times 10^{7} M_{\odot}$ & $1.475 \times 10^{11}$ & $6.5 \times 10^{45}$ & 0.01135 \\
\hline 15 & $6 \times 10^{7} M_{\odot}$ & $1.770 \times 10^{11}$ & $7.8 \times 10^{45}$ & 0.01362 \\
\hline 16 & $7 \times 10^{7} M_{\odot}$ & $2.065 \times 10^{11}$ & $9.1 \times 10^{45}$ & 0.01589 \\
\hline 17 & $8 \times 10^{7} M_{\odot}$ & $2.360 \times 10^{11}$ & $10.4 \times 10^{45}$ & 0.01816 \\
\hline 18 & $9 \times 10^{7} M_{\odot}$ & $2.655 \times 10^{11}$ & $11.7 \times 10^{45}$ & 0.02043 \\
\hline 19 & $1 \times 10^{8} M_{\odot}$ & $2.950 \times 10^{11}$ & $1.3 \times 10^{46}$ & 0.02277 \\
\hline 20 & $2 \times 10^{8} M_{\odot}$ & $5.950 \times 10^{11}$ & $2.6 \times 10^{46}$ & 0.04554 \\
\hline 21 & $3 \times 10^{8} M_{\odot}$ & $8.850 \times 10^{11}$ & $3.9 \times 10^{46}$ & 0.06831 \\
\hline 22 & $4 \times 10^{8} M_{\odot}$ & $11.80 \times 10^{11}$ & $5.2 \times 10^{46}$ & 0.09108 \\
\hline 23 & $5 \times 10^{8} M_{\odot}$ & $14.75 \times 10^{11}$ & $6.5 \times 10^{46}$ & 0.11385 \\
\hline 24 & $6 \times 10^{8} M_{\odot}$ & $17.70 \times 10^{11}$ & $7.8 \times 10^{46}$ & 0.13662 \\
\hline 25 & $7 \times 10^{8} M_{\odot}$ & $20.65 \times 10^{11}$ & $9.1 \times 10^{46}$ & 0.15939 \\
\hline 26 & $8 \times 10^{8} M_{\odot}$ & $23.60 \times 10^{11}$ & $10.4 \times 10^{46}$ & 0.18216 \\
\hline 27 & $9 \times 10^{8} M_{\odot}$ & $26.55 \times 10^{11}$ & $11.7 \times 10^{46}$ & 0.20493 \\
\hline 28 & $1 \times 10^{9} M_{\odot}$ & $29.50 \times 10^{11}$ & $1.3 \times 10^{47}$ & 0.22777 \\
\hline 29 & $2 \times 10^{9} M_{\odot}$ & $59.50 \times 10^{11}$ & $2.6 \times 10^{47}$ & 0.45552 \\
\hline 30 & $3 \times 10^{9} M_{\odot}$ & $88.50 \times 10^{11}$ & $3.9 \times 10^{47}$ & 0.68328 \\
\hline 31 & $4 \times 10^{9} M_{\odot}$ & $93.28 \times 10^{11}$ & $5.2 \times 10^{47}$ & 0.7207 \\
\hline 32 & $5 \times 10^{9} M_{\odot}$ & $118.0 \times 10^{11}$ & $6.5 \times 10^{47}$ & 0.91104 \\
\hline 33 & $6 \times 10^{9} M_{\odot}$ & $147.5 \times 10^{11}$ & $7.8 \times 10^{47}$ & 1.1388 \\
\hline
\end{tabular}

\section{Result and Discussion}

When we have calculated the efficiency for $R_{\text {stable }}=9 \mathrm{GM} / \mathrm{c}^{2}, \quad R_{\text {stable }}=6 \mathrm{GM} / \mathrm{c}^{2}, \quad R_{\text {stable }}=2 \mathrm{GM} / \mathrm{c}^{2}$, $R_{\text {stable }}=1.2 \mathrm{GM} / \mathrm{c}^{2}$ and $R_{\text {stable }}=\mathrm{GM} / \mathrm{c}^{2}$ on the basis of equation (2), the radiative efficiency $(\eta)$ is given by 0.11 ,
$0.17,0.50,0.83$ and 1.00 respectively. Narayan (2005), Xie \& Yuan (2012) and Chan (2013) have discussed in their papers that for non-rotating

black holes, $\eta=0.057$, whereas a co-rotating disk around a maximally-rotating black holes $\left(a_{*}=1\right)$ has a much higher efficiency, $\eta=0.42$. Pant et al. (2010) showed luminosity of quasars the black holes of mass $10^{8} M_{\odot}$ is $10^{47} \mathrm{erg} / \mathrm{sec}$. giving the radiative efficiency equal to 0.1752 . In the present work, we have calculated radiative efficiency $\eta$ for different test black holes existing in AGN. Our calculation shows that the radiative efficiency $\eta$ lies between 0.00022 and 0.91104 corresponding to the mass ranging from $10^{6} M_{\odot} t o 5 \times 10^{9} M_{\odot}$ in AGN. From the expression of radiative efficiency and data observed from the table 1 , it is clear that the radiative efficiency of black holes increases with increasing the mass of black holes. Assuming an isotropic, spherically symmetric system, Sargent et al. detected a central dark mass $\sim 5 \times 10^{9} M_{\odot}$ within the inner 110pc of M87 (Sargent, 1978).

Corresponding to the mass of black hole equal to $6 \times 10^{9} M_{\odot}$, the radiative efficiency is 1.388 indicating that the efficiency is greater than $100 \%$, which is not possible. From theoretical discussion with the help of the equation (2), the maximum radiative efficiency is 1.00 . This shows the existence of central dark mass can not exceed to the mass to $5 \times 10^{9} M_{\odot}$ within the inner 110pc of M87. When we compare the radiative efficiency of black holes of mass $10^{8} M_{\odot}$ with the same mass of quasars (Pant \& Tiwari, 2010), we obtain that the radiative efficiency of black holes is 0.02277 and that for quasars is 0.1752 . This data shows that the radiative efficiency of black holes is very-very small for the same mass of quasars, because total core part of quasars is converted into nuclear energy, but in the case of black holes, total core part is not converted into nuclear energy.

\section{Conclusion}

In course of the present research work, we can draw the following conclusions such as:

(1) The mass of black holes never be greater than $5 \times 10^{9} M_{\odot}$ which agrees with the Experimental result (Sargent et al.1978).

(2) The radiative efficiency $(\eta)$ of black hole is lower for lower mass and vice-versa.

(3) The radiative efficiency $(\eta)$ of black hole is smaller than the same mass of quasars.

\section{Acknowledgement}

The authors are obliged to their teacher Dr. Gopi Kant Jha, Former Head \& Prof. of Physics, L.N.M.U Darbhanga for his inspiration and motivation. 


\section{References}

[1] B. Mckernan, K.E.S. Ford and C. S. Reynolds: Black hole mass, Host galaxy classification and AGN activity. arXiv: 10054907vi[astro-ph].26 May 2010.

[2] L. Ferrarese \& H. Ford: Super massive Black Holes in Galactic Nuclei: Past Present \& Future Research, Space Science Reviews 116:523-624, 2005.

[3] A. C. Fabian: "Broad iron lines in AGN and X-ray binaries." Astrophysics and space Science 300: 97-105, 2005, DOI: 10.1007/s10509-005-1203-x.

[4] Neeraj Pant \& B.C. Tiwari: "Horizon- free gravitational collapse of radiating fluid sphere." Astrophys and space science (2010).

[5] R. Narayan: Black Holes in Astrophysics, New Journal Physics, Vol. 7, No.1, 2005, pp1-31.

[6] Fu-Guo Xie and Feng Yuan: The Radiative Efficiency of Hot Accretion Flows. arXiv :1207.3113v2[astro-ph.] 3 Sept. 2012.

[7] M.H. Chan: Shaping the relation between the mass of the super massive black holes and the velocity dispersion of galactic bulges, Astrophys Space Science. DOI: 10.1007/s 10509-013-1373-x, May 2013, Volume 345, issue 1, pp195-198.

[8] D. Mahto, V. Prakash, B.K.Singh, K.M. Singh: Change in entropy of Non-spinning black holes w.r.t. the radius of event horizon in XRBs, Astrophys SpaceSci. Doi 10.1007/s10509-012-1219-y (2012).

[9] Mahto, D, Nadeem, M. D., Prasad, U., Kumar, A.\& Singh, K.M.: Change in entropy of Non-spinning black holes w.r.t. the radius of event horizon in AGN, Journal of Modern Physics, doi:10.4236/jmp.2013.43043, March 2013.

[10] W.L.W. Sargent. et al. :Dynamical Evidence for a Central Mass Concentration in the Galaxy M87, The Astrophysical Journal, Vol. 221, 1978, p.731.

[11] Astro 3830: Spring 2004. 